\title{
DISCUSSÃO DO SISTEMA DE CONTROLE DE ATITUDE DE UM VEÍCULO LANÇADOR
}

\author{
Adolfo Graciano da Silva*, Alexandro Garro Brito*, Waldemar de Castro leite Filho* \\ * Instituto de Aeronáutica e Espaço \\ Pça Mal. Eduardo Gomes 50, vila das Acácias. CEP: 12228-904 \\ São José dos Campos-SP Brazil
}

Emails: adolfo.graciano@gmail.com, alegbrito2@gmail.com, waldemarwclf@iae.cta.br

\begin{abstract}
Resumo- Neste trabalho são discutidos os principais conceitos e requisitos utilizados durante o projeto da malha de controle de atitude de um veículo lançador a partir da análise da dinâmica simplificada do veículo. A análise é dividida em duas partes, primeiramente o veículo é tratado como um corpo rígido e um controlador PID é apresentado. Em seguida o veículo é considerado um corpo flexível e dois métodos de estabilização dos modos flexão (ganho ou por fase) são apresentados. Além disso, um sistema de controle de atitude é projetado
\end{abstract} considerando os conceitos discutidos.

Palavras-chave - Controle de Atitude, Veículo Lançador, Margens de Estabilidade, Modos de Flexão

\begin{abstract}
In this paper some concepts and requirements about the launch vehicle attitude control system are discussed from the analysis of the simplified vehicle dynamics. The analysis is split in two parts, first of all the vehicle is treated as rigid body and a PID controller is presented. Then the vehicle is treated as a flex-body and two methods of bending mode stabilization are shown. Moreover, an attitude control system is designed based on concepts discussed.
\end{abstract}

Keywords - Attitude Control, Launch Vehicle, Stability Margins, Bending Modes

\section{Introdução}

O projeto do sistema de controle de atitude de um veículo lançador representa um grande desafio, pois a dinâmica do veículo é regida por um sistema de equações não-lineares, acopladas e variantes no tempo. Tais equações não possuem solução analítica na maioria das situações. Apesar de soluções numéricas serem possíveis, essas não oferecem ao projetista um entendimento direto de algumas características do veículo e, além disso, não permitem a análise e projeto utilizando a teoria clássica de controle. Para contornar esse problema, são realizadas algumas suposições e, em seguida, as equações são linearizadas em torno da trajetória de referência. Obtêm-se então um sistema de equações lineares desacopladas, mas variantes no tempo. A fim de permitir a análise através de ferramentas clássicas de controle tais como diagramas de resposta em frequência, lugar das raízes e etc, é assumido que os parâmetros relacionados ao veículo não variam durante um curto período de tempo, técnica essa conhecida como dinâmica congelada (Kadam, 2009). Esse procedimento é aplicado durante todo o voo, obtendo-se um sistema de equações lineares e invariantes no tempo para cada instante analisado. De posse dessas equações um sistema de controle é projetado para garantir a estabilidade e desempenho necessários durante todo o voo.

O sistema de controle de atitude de vários veículos lançadores é composto por um controlador PID para o corpo rígido e alguns compensadores para estabilizar os modos de flexão. Em alguns casos, uma malha adicional de alívio de carga ae- rodinâmica (load-relief) é utilizada para minimizar os esforços estruturais sofridos pelo veículo durante a ascensão na atmosfera. Essa arquitetura tem sido utilizada desde os anos 60 e sua eficácia foi comprovada em vários voos (Greensite, 1970). Apesar de novas técnicas de controle terem surgido desde os anos 70 , os veículos lançadores mais modernos ainda utilizam o clássico PID para a estabilização da malha de atitude (Hall et al., 2013). O Veículo Lançador de Satélites (VLS) brasileiro, também utiliza-se de um controlador PID e filtros para estabilização da malha de atitude (Leite Filho, 2000).

Nesse trabalho algumas características de suma importância para o projeto do sistema de controle de atitude serão abordadas, tais como: as margens de estabilidade do corpo rígido, modos de flexão e rastreamento do sinal de referência. Também será estudado como os ganhos do controlador e os polos dominantes afetam essas características. Em seguida será apresentado um estudo de caso.

\section{Visão geral do sistema de controle de atitude de um veículo lançador}

A Figura 1 contém os principais blocos do sistema de atitude de um veículo lançador. Tal sistema possui um algoritmo de guiamento, que utiliza-se das medidas de sensores inerciais (acelerômetros e giros) com a finalidade de obter a posição atual do veículo para o cálculo das correções necessárias( comandos de atitude) para mantê-lo na trajetória desejada. O controlador de atitude tem como objetivo rastrear o sinal em atitude advindo do guiamento o mais rápido possível. Além disso, o 


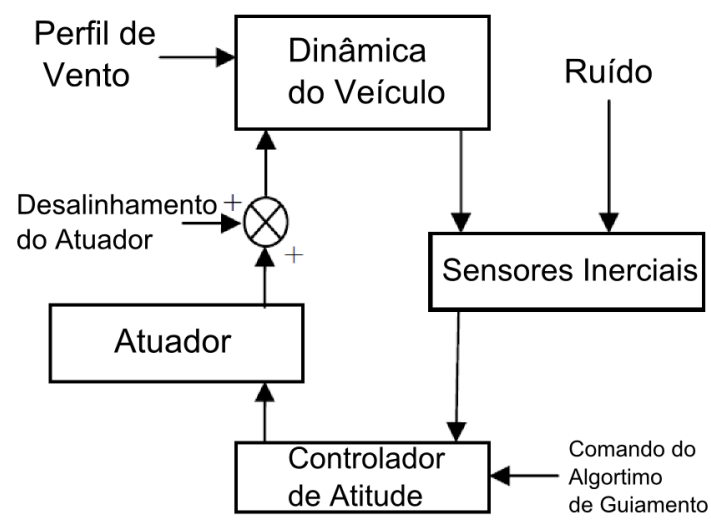

Figura 1: Sistema de controle de atitude de um veículo lançador (Renault and Saunois, 2008)

sistema de controle deve ser capaz de rejeitar as perturbações advindas do desalinhamento do atuador e do vento.

A seguir, são apresentadas as equações que regem a dinâmica do atuador e do veículo respectivamente. A função de transferência do atuador utilizada nesse trabalho é dada por

$$
G_{a}(s)=\frac{K_{A}}{s+K_{A}}
$$

onde $K_{A}$ é a banda passante. As equações que regem a dinâmica do veículo são obtidas através de uma perturbação linear em torno da trajetória de referência (Greensite, 1970). Essas escritas na forma de espaço de estado

$$
\left[\begin{array}{c}
\dot{\theta} \\
\ddot{\theta} \\
\dot{w}
\end{array}\right]=\left[\begin{array}{ccc}
0 & 1 & 0 \\
0 & 0 & \frac{\mu_{\alpha}}{U_{0}} \\
-g \cos \theta_{0} & U_{0} & -\frac{Z_{\alpha}}{U_{0}}
\end{array}\right]\left[\begin{array}{c}
\theta \\
\dot{\theta} \\
w
\end{array}\right]+\left[\begin{array}{c}
0 \\
\mu_{\beta} \\
Z_{\delta}
\end{array}\right] \delta+\left[\begin{array}{c}
0 \\
\mu_{\alpha} \\
-Z_{\alpha}
\end{array}\right] \alpha_{w}
$$

onde $\theta$ é o ângulo de atitude perturbado, $\theta_{0}$ é atitude de referência na qual as equações foram linearizadas, $\alpha_{w}$ é o ângulo de ataque causado pelo vento, $g$ é gravidade, $U_{0}$ é a velocidade longitudinal do veículo e $w$ é a velocidade perturbada paralela ao eixo $Z_{b}$ do veículo, que é perpendicular ao eixo longitudinal do veículo. Ainda temos,

$$
\begin{aligned}
& \mu_{\beta}=\frac{T_{c} l_{c}}{I_{y y}} ; \quad \mu_{\alpha}=\frac{L_{\alpha} l_{\alpha}}{I_{y y}} ; \\
& Z_{\alpha}=\frac{L_{\alpha}}{m_{T}} ; \quad Z_{\beta}=\frac{T_{c}}{m_{T}} ;
\end{aligned}
$$

onde $T_{c}$ é a força de empuxo, $L_{\alpha}$ é a força aerodinâmica que age no centro de pressão do veículo, $l_{c}$ é a distância do centro de gravidade ao ponto de aplicação da força, $l_{\alpha}$ é a distância do centro de gravidade até o centro de pressão, $m_{T}$ é a massa total do veículo e $I_{y y}$ o momento de inércia em torno do eixo $y$ do veículo.

Dadas as equações acima, a função de transferência da planta a partir da variável de controle $\delta$ para a saída $\theta$ é

$$
G_{c r}=\frac{\theta(s)}{\delta(s)}=\frac{\mu_{\beta} s+\frac{\mu_{\beta} Z_{\alpha}}{U_{0}}+\frac{\mu_{\alpha} Z_{\beta}}{U_{0}}}{s^{3}+\frac{Z_{\alpha}}{U_{0}} s^{2}-\mu_{\alpha} s+\frac{\mu_{\alpha} g \cos \theta_{0}}{U_{0}}}
$$

Em regiões de alta pressão dinâmica a equação anterior pode ser aproximada por

$$
G_{c r}=\frac{\theta(s)}{\delta(s)}=\frac{\mu_{\beta}}{s^{2}-\mu_{\alpha}} .
$$

A equação anterior modela somente a dinâmica do corpo rígido, não levando em consideração os efeitos relacionados à flexão do veículo. Posteriormente, serão apresentadas as equações que regem a dinâmica do veículo lançador considerando tais modos de flexão. A dinâmica do sensor será desprezada nas análises preliminares, pois considerase que esse possua uma banda passante muito maior que qualquer dinâmica de interesse do veículo.

\section{Análise do sistema de controle de atitude}

Dado o diagrama em blocos da Figura 2 e analisando as equações simplificadas do corpo rígido, obtêm-se importantes características do sistema de controle de atitude de um veículo lançador. Suponha que a dinâmica do atuador e do veículo sejam dadas por (1) e (4) respectivamente e, que o termo $K_{I}$ do controlador é nulo. Logo, a equação em malha fechada do sistema é dada por

$$
G_{F}=\frac{b_{0}}{s^{3}+a_{2} s^{2}+a_{1} s+a_{0}}
$$

e

$b_{0}=K_{A} K_{p} \mu_{\beta}$

$a_{2}=K_{A}$

$a_{1}=K_{A} K_{p} K_{d} \mu_{\beta}-\mu_{\alpha}$

$a_{0}=K_{A}\left(K_{p} \mu_{\beta}-\mu_{\alpha}\right)$

Aplicando-se o critério de estabilidade RouthHurwitz, as seguintes relações são obtidas.

$$
K_{p} \mu_{\beta}-\mu_{\alpha}>0
$$

e

$$
K_{d}>1 / K_{A}
$$

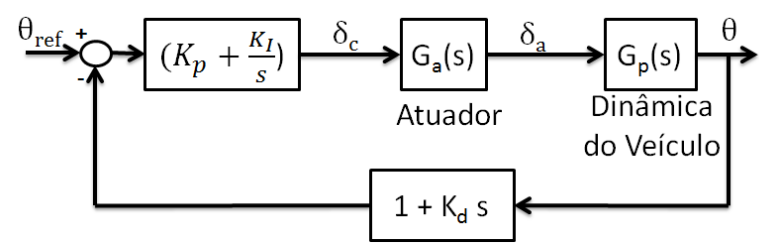

Figura 2: Arquitetura PID 
A equação (6) representa à condição de estabilidade estática, isto é, o momento controle deve ser maior que o momento aerodinâmico. Já (7) refere-se à condição de estabilidade dinâmica, ou seja, o avanço de fase proporcionado pelo termo derivativo deve ser maior que o atraso de fase proporcionado pelo atuador. Note que, a partir dessas equações, o ganho proporcional do controlador pode ser aumentando indefinidamente. Entretanto, haverá um limite superior assim que as dinâmicas de ordem elevada(filtros para estabilizar os modos de flexão, sensores e etc) forem consideradas. Outro limitante ao incremento do ganho $K_{p}$ advém da saturação do atuador. A Figura 3 apresenta um gráfico qualitativo das margens de estabilidade típicas de um veículo lançador. A área em azul representa uma região proibida para a garantia de boas margens de estabilidade para o sistema de controle de atitude. Vale salientar que boas margens de estabilidade são necessárias no projeto do sistema de controle de atitude, devido às grandes incertezas paramétricas de um veículo lançador.

Nessa seção foram apresentadas as margens de estabilidade relacionadas apenas ao corpo rígido. Outras margens relacionadas aos modos de flexão serão descritas na seção 4 .

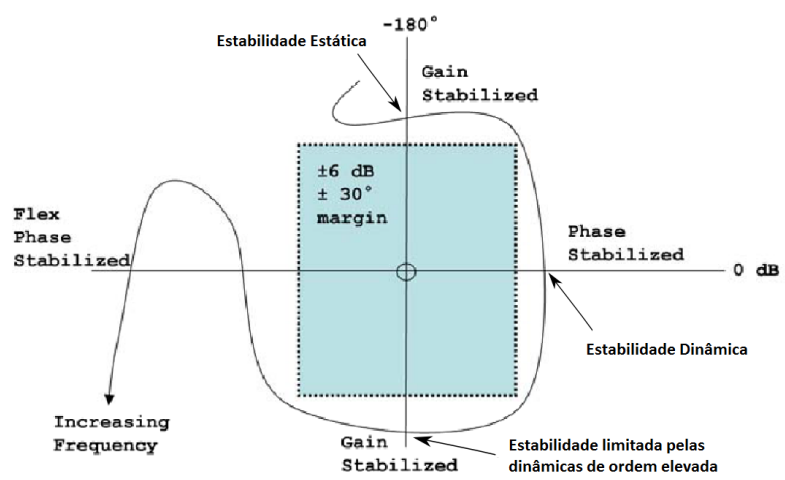

Figura 3: Carta de Nichols contendo as margens de estabilidade do corpo rígido (Whorton et al., 2007)

Outra caraterística importante que o sistema de controle de atitude deve possuir é ser capaz de rastrear o comando de atitude advindo do algoritmo de guiamento com o mínimo erro possível. Da teoria de controle linear é sabido que o posicionamento dos polos dominantes determina em grande parte a resposta transitória do sistema de controle de atitude, além da resposta em regime permanente. Suponha um controlador PD, no qual os ganhos sejam selecionados de forma a alocar os polos dominantes, formando um par complexo conjugado do tipo

$$
D(s)=\left(s^{2}+2 \zeta \omega_{n} s+\omega_{n}^{2}\right)(s+p)
$$

onde $\omega_{n}$ e $\zeta$ são respectivamente a frequência natural de oscilação e o amortecimento associados aos polos dominantes do sistema. Comparando os coeficientes da equação anterior com a equação (5) e realizando algumas manipulações algébricas, são obtidas as equações

$$
K_{p} \mu_{\beta}-\mu_{\alpha}=\omega_{n}^{2}\left(1-\frac{2 \zeta \omega_{n}}{K_{A}}\right)
$$

e

$$
K_{d}=\frac{\omega_{n}^{2}+2 \zeta \omega_{n}\left(K_{A}-2 \zeta \omega_{n}\right)}{K_{A} K_{p} \mu_{\beta}}+\frac{\mu_{\alpha}}{K_{A} K_{p} \mu_{\beta}}
$$

Note que o lado esquerdo de (9) é exatamente igual à estabilidade estática. Logo, se os polos dominantes da equação são especificados, indiretamente uma das margens de estabilidade do sistema será definida. Então, os ganhos $K_{p}$ e $K_{d}$ podem ser calculados a partir dos polos dominantes.

Durante a fase de voo atmosférica, a maioria dos veículos lançadores seguem um perfil de atitude pré-determinado, advindo da otimização da trajetória utilizando a estratégia do gravity turn (Greensite, 1970). O perfil de atitude criado a partir dessa otimização pode ser bem aproximado por segmentos de retas durante a ascensão do foguete na fase atmosférica. Dado um sinal de referência da forma $r(t)=\dot{\theta}_{c} t$, onde $\dot{\theta}_{c}$ é a variação do sinal de comando, a equação do erro em regime permanente à rampa é dada por

$$
\begin{gathered}
e_{s s}(t)=\dot{\theta}_{c} \frac{-\mu_{\alpha}}{K_{p} \mu_{\beta}-\mu_{\alpha}} t+\dot{\theta}_{c} \frac{K_{d} K_{p}^{2} \mu_{\beta}^{2}}{\left(K_{p} \mu_{\beta}-\mu_{\alpha}\right)^{2}} \\
e_{s s}(t)=\dot{\theta}_{c} \frac{K_{I} K_{d} \mu_{\beta}-\mu_{\alpha}}{K_{I} \mu_{\beta}}
\end{gathered}
$$

para um controlador PD e PID respectivamente. Note em (11) que a taxa de variação do erro em regime estacionário está diretamente relacionada com a estabilidade estática e com o coeficiente de momento aerodinâmico. No caso em que o controlador PID é utilizado o erro em regime permanente é limitado. Porém, se escolhidos adequadamente como

$$
K_{I} K_{d}=\frac{\mu_{\alpha}}{\mu_{\beta}}
$$

obtém-se erro nulo (de Souza and Leite Filho, 2011). Os resultados obtidos para o erro em regime permanente são apenas qualitativos. Visto que o sistema é não-linear e variante no tempo. Logo, esses resultados servem apenas como uma referência para o projeto do controlador indicando tendências do sistema.

Durante esse processo, é visível que o aumento da banda passante do sistema de controle de atitude melhora o rastreio. Entretanto, a banda passante do controlador de atitude não pode ser aumentada indefinidamente, pois, como será visto na próxima seção, deve haver uma separação mínima entre a banda passante do controlador e o primeiro 
modo de flexão para que seja possível a estabilização deste. Sendo assim, a banda passante do corpo rígido deve ser escolhida como sendo a menor possível, para que se atinja os requisitos de rastreio e proporcione a maior separação em frequência possível entre o corpo rígido e os modos de flexão.

\section{Modos de flexão}

Para descrever bem a dinâmica de um veículo lançador é necessário considerar a dinâmica dos modos de flexão do veículo (Greensite 1970). Os modos de flexão podem ser representados pela função de transferência

$$
G_{f_{i}}(s)=\frac{\theta_{f_{i}}(s)}{\delta(s)}=\frac{K_{f_{i}}}{s^{2}+2 \zeta_{f_{i}} \omega_{f_{i}}+\omega_{f_{i}}^{2}}
$$

onde $\omega_{f_{i}}, \zeta_{f_{i}}$ e $K_{f_{i}}$, são respectivamente, a frequência natural de oscilação, o amortecimento e o ganho associado ao enésimo modo de flexão. Observando a Figura 4, note que os modos de flexão são excitados pelo atuador e sua interação com o sistema de controle de atitude é dada através dos sensores de velocidade e posição. Assim há uma interação entre a estrutura e o controle, podendo causar a instabilidade da malha. A interação dos modos de flexão com a malha de atitude é fortemente influenciada pela localização dos sensores de atitude (Kadam, 2009).

A estabilização dos modos de flexão é considerada por muitos o maior desafio no projeto do sistema de controle de atitude, pois o sistema deve ser capaz de estabilizar os modos flexão mesmo na presença de incertezas paramétricas e sem degradar o desempenho do corpo rígido. Uma das

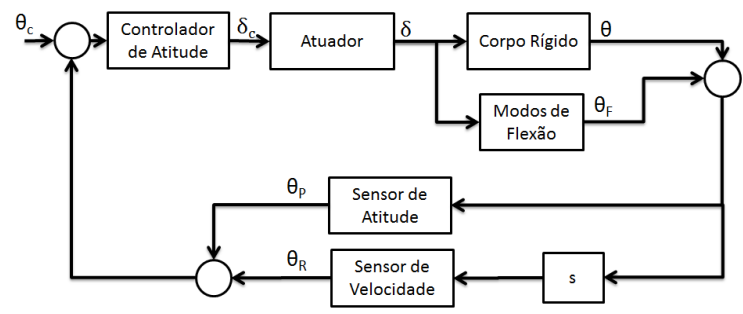

Figura 4: Sistema de controle considerando os modos de flexão

técnicas normalmente utilizadas para a estabilização dos modos de flexão é a estabilização por ganho. Na qual filtros são adicionados na malha impedindo que o sinal de comando atue na frequência de flexão (Kadam, 2009). Entretanto, para que seja possível a utilização dessa técnica, é necessário uma separação mínima entre a frequência de controle do corpo rígido e a frequência de oscilação do primeiro modo de flexão, pois a adição desse tipo de filtro atrasa a fase na região de sintonia. Muitas vezes as frequências de flexão previstas através de softwares de elementos finitos encontram-se fora daquelas observadas em voo. Logo, o projetista deve se atentar a esse fato e projetar um filtro capaz de estabilizar o modo de flexão mesmo em caso de incertezas. Um requisito utilizado em projetos de veículos lançadores para garantir a robustez do sistema a tais variações, é garantir que o ganho do modo de flexão, não ultrapasse um determinado valor em $\mathrm{dB}$, normalmente entre 6 a $10 \mathrm{~dB}$ na região de variação de frequência do modo de flexão(Jang et al., 2010). Tal requisito pode ser observado na Figura 5. Existem

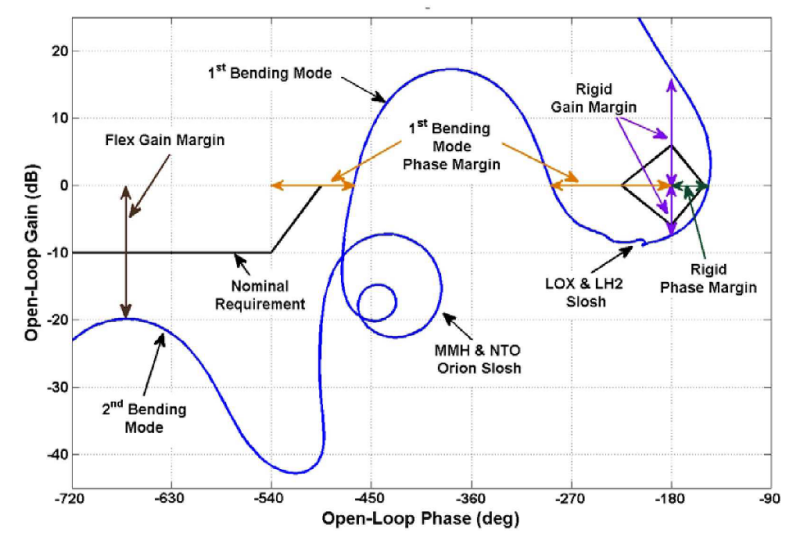

Figura 5: Definição das margens de estabilidade dos modos de flexão (Jang et al., 2010)

casos nos quais a frequência do primeiro modo de flexão está muito próxima do corpo rígido. Logo, a tentativa de estabilização por ganho irá prejudicar consideravelmente as margens de estabilidade do corpo rígido. Quando isso ocorre, é necessário realizar a estabilização por fase (Kadam, 2009). Esse tipo técnica ao contrário da estabilização por ganho implica que o atuador responda na frequência do primeiro modo de flexão. Note na Figura 5, que o primeiro modo de flexão corta o $0 \mathrm{~dB}$ duas vezes, logo é necessário prover fases adequadas nessas duas regiões. O requisito utilizado é $45^{\circ}$ de margem de fase (Jang et al., 2010). Além disso, recomenda-se a estabilização por fase somente do primeiros modo de flexão, visto que a incerteza nas altas frequências impossibilita assegurar a fase necessária para a estabilização do mesmo.

\section{Estudo de Caso}

Para exemplificar os conceitos discutidos até o momento, será projetado o sistema de controle de atitude considerando apenas o instante de voo no qual o veículo está sob o maior estresse aerodinâmico. Este instante é definido como a região de máxima pressão dinâmica. O projeto será dividido em duas partes: primeiramente serão calculados os ganhos do controlador para o corpo rígido, e, em seguida, serão calculados os filtros para a estabilização dos modos de flexão.

Os parâmetros relacionados ao instante de 
voo mencionado, considerando apenas o primeiro e segundo modos de flexão, são dados na tabela abaixo. A estrutura do controlador utilizado é

\begin{tabular}{|c|c|c|c|c|c|}
\hline \multicolumn{6}{|c|}{ Tabela de Parâmetros } \\
\hline$\mu_{\alpha}$ & $\mu_{\beta}$ & $Z_{\alpha}$ & $Z_{\beta}$ & $g$ & $U_{0}$ \\
4.16 & 7.21 & 48.95 & 19.92 & 9.78 & 597 \\
\hline$\omega_{f_{1}}$ & $\omega_{f_{2}}$ & $\zeta_{f_{1}}$ & $\zeta_{f_{2}}$ & $K_{f_{1}}$ & $K_{f_{2}}$ \\
30 & 79 & 0.002 & 0.002 & -15 & 10 \\
\hline
\end{tabular}

Tabela 1: Tabela contendo os valores dos parâmetros relacionados ao instante de máxima pressão dinâmica

apresentada na Figura 6. A dinâmica da planta é dada por

$$
G_{p}(s)=G_{c r}+G_{f_{1}}+G_{f_{2}}
$$

e do atuador é dada em (1). Os ganhos do controlador podem ser obtidos através de diversas metodologias, desde algoritmos de otimização a métodos analíticos (Brito et al., 2006). Nesse trabalho será utilizado o método analítico na qual os ganhos são calculados a partir da especificação dos polos dominantes do sistema $((9)$ e $(10))$.

Escolhendo os parâmetros dos polos dominantes como sendo $\omega_{n}=3.4$ e $\zeta=0.95$, obtêm-se $K_{p}=1.82$ e $K_{d}=0.44$. Esses dois valores proporcionam margens de fase e ganho adequadas para o corpo rígido, respeitando a restrição da frequência de cruzamento de ganho, além de prover uma resposta rápida e adequada ao sistema de controle de atitude.

O segundo passo é estabilizar os modos de flexão. Esse passo é iterativo e depende muito da experiência do projetista. Mas, recentemente têm sido utilizados algoritmos de otimização numérica para sintonizar os filtros a fim de maximizar alguns requisitos da malha de controle (Jang et al., 2010) e (Renault and Saunois, 2008).

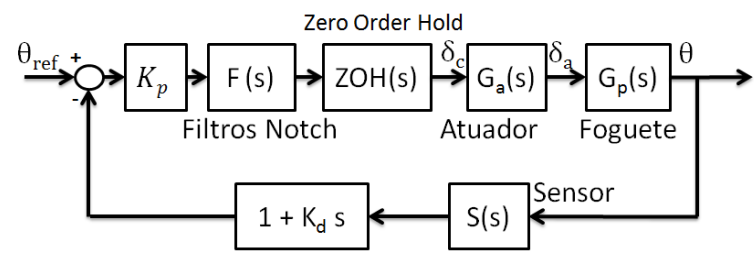

Figura 6: Estrutura contendo os filtros Notch

No estudo de caso apresentado nesse artigo foram utilizados dois filtros notch de fase mínima no canal direto, para estabilizar o primeiro e o segundo modo de flexão (Figura 6) . A equação do filtro notch é dada por

$$
F_{N}(s)=\left(\frac{\omega_{d}}{\omega_{n}}\right)^{2} \frac{s^{2}+2 \zeta_{n} \omega_{n} s+\omega_{n}^{2}}{s^{2}+2 \zeta_{d} \omega_{d} s+\omega_{d}^{2}}
$$

Cada filtro foi sintonizado heuristicamente, a fim de proporcionar a estabilização dos modos de flexão e a mínima degradação do corpo rígido. Os parâmetros de sintonia dos filtros são apresentados na tabela abaixo.

\begin{tabular}{|c|c|c|c|c|}
\hline \multicolumn{5}{|c|}{ Coeficientes dos Filtros } \\
\hline & $\omega_{n}$ & $\omega_{d}$ & $\zeta_{n}$ & $\zeta_{d}$ \\
\hline$F_{N 1}$ & 38 & 38 & 0.02 & 1 \\
\hline$F_{N 2}$ & 80 & 80 & 0.02 & 1 \\
\hline
\end{tabular}

Tabela 2: Tabela dos coeficientes relacionados aos filtros Notch

Para explorar os conceitos discutidos nesse artigo considere primeiramente somente o filtro Notch 1 inserido na malha e todas as outras dinâmicas, exceto o atraso devido a computação digital $(\mathrm{ZOH})$. A partir disso é obtido o diagrama de Nichols da Figura 7. Note que ambos os modos de flexão estão estabilizados por fase e aparentemente possuem boas margens de estabilidade. Entretanto, como já foi dito na seção 4 , não é recomendado estabilizar os modos de flexão superiores dessa maneira.

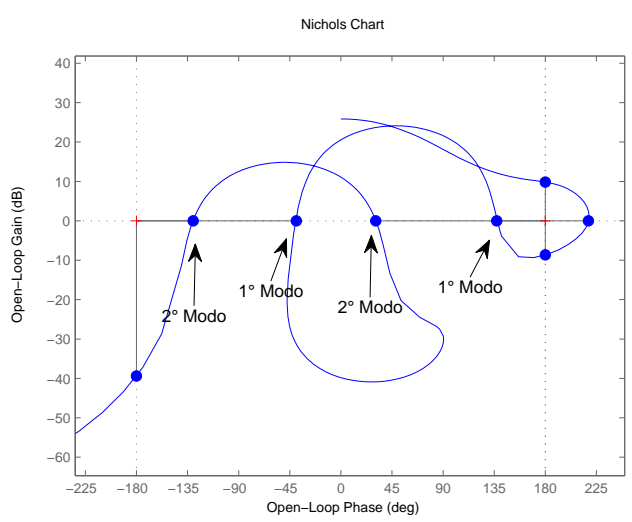

Figura 7: Diagrama de Nichols considerando somente o filtro Notch 1 e sem o $\mathrm{ZOH}$

$\mathrm{Na}$ Figura 8 encontra-se o diagrama de Nichols acrescido da dinâmica da computação digital. Note que apesar das boas margens de estabilidade do segundo modo Figura 7, o sistema se tornou instável. Isso porque, o pequeno atraso da computação digital já foi o suficiente para consumir toda essa margem.

A Figura 9 contém o diagrama de Nichols do sistema completo acrescido do filtro Notch 2. Veja que o sistema é estável e cumpre quase todos os requisitos de estabilidade. As margens de estabilidade do corpo rígido foram um pouco degradas pela inserção dos filtros na malha. Note que ao reduzir a banda passante do corpo rígido escolhendo um polo dominante um pouco mais lento, as margens de estabilidade críticas (margem de fase e a margem de ganho superior) do corpo rígido foram melhoradas.

Esses resultados foram satisfatórios para um intervalo de tempo. Entretanto, como o sistema é 


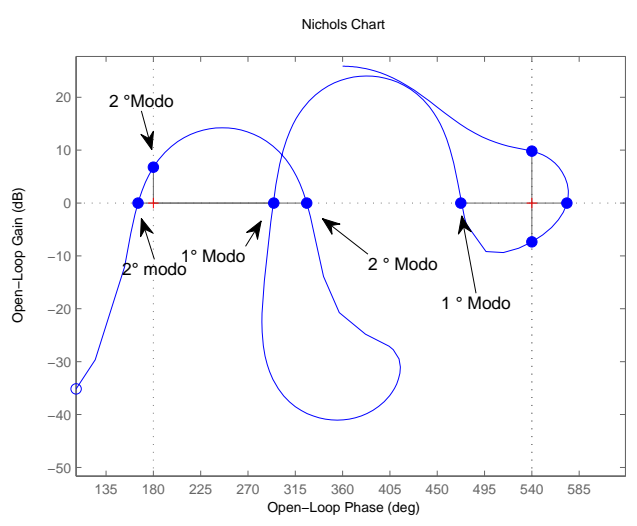

Figura 8: Diagrama de Nichols considerando todas as dinâmicas, exceto o filtro Notch 2

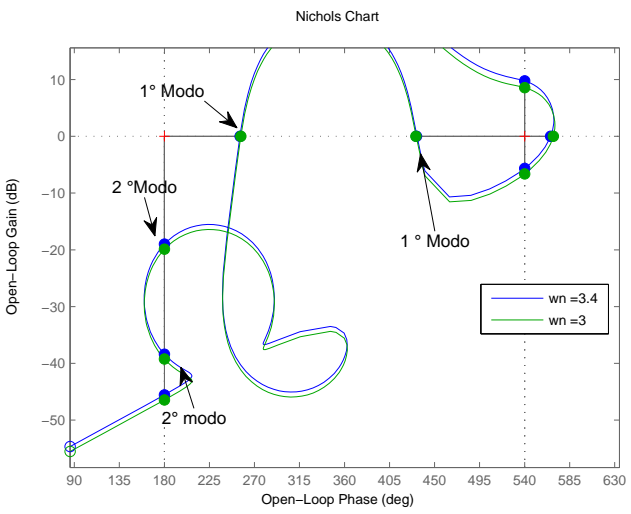

Figura 9: Diagrama de Nichols considerando todas as dinâmicas e os filtros Notch

variante no tempo, esse controlador deve ser testado para todos os outros intervalos de tempo. Normalmente, é necessário realizar um escalonamento dos ganhos do controlador para garantir as margens de estabilidade e boa resposta dinâmica durante todo o voo (Brito et al., 2006). Em seguida, é necessário realizar uma simulação de 6 graus de liberdade contendo todas as dinâmicas não consideradas no projeto do controlador, os perfis de vento esperados durante o voo na atmosfera, além das não-linearidades do atuador, com a finalidade de garantir o desempenho e estabilidade do sistema de controle de atitude.

\section{Conclusão}

Neste trabalho foram apresentados os conceitos básicos utilizados no projeto do controlador de atitude de um veículo lançador, obtidos a partir da análise linear da dinâmica simplificada. Além disso, foi visto que a banda passante máxima do corpo rígido é limitada principalmente pelo primeiro modo de flexão. Na análise dos modos de flexão foram mostradas duas estratégias diferentes, a estabilização por ganho e por fase. Quando possível a estabilização por ganho deve ser usada, pois evita que o atuador trabalhe nas frequências dos modos de flexão, além de dar mais robustez ao sistema. E finalmente, foi apresentado um estudo de caso no qual foram aplicados os conceitos discutidos nesse artigo.

\section{Agradecimentos}

Este trabalho é financiado pelo SIAFINEP/Fundep.

\section{Referências}

Brito, A. G., Leite Filho, W. C. and Ramos, F. O. (2006). Approach comparison for controller design of a launcher, ESA Conference on Guidance, Navigation and Control Systems, Loutraki, Greece, 17-20 October 2005.

de Souza, J. P. and Leite Filho, W. C. (2011). Estudo comparitivo de arquiteturas de controlador pid para veículos aerodinamicamente instáveis, DINCON .

Greensite, A. L. (1970). Analysis and design of space vehicle flight control systems, Spartan Books.

Hall, C., Lee, C., Jackson, M., Whorton, M., West, M., Brandon, J., Hall, R. A., Jang, J., Bedrossian, N., Compton, J. and Rutherford, C. (2013). Ares i flight control system overview, AIAA .

Jang, J.-W., Alaniz, A., Hall, R., Bedrossian, N., Hall, C., Ryan, S. and Jackson, M. (2010). Ares i flight control system design, AIAA .

Kadam, N. V. (2009). Practical Design of Flight Control Systems for Launch Vehicles and Missiles, Allied Publishers Pvt.

Leite Filho, W. C. (2000). Control system of brazilian launcher, $4^{\text {th }}$ ESA Intnl. Conf. on Spacecraft, GNC.

Renault, C. and Saunois, P. (2008). Launchers control architecture and synthesis with analytical loop shaping, th $^{\text {th }}$ International ESA Conference on Guidance, Navigation and Control Systems .

Whorton, M. S., Hall, C. E. and Cook, S. A. (2007). Ascent flight control and structural interaction for the ares-i crew launch vehicle, $48^{\text {th }}$ AIAA Structures, Structural Dynamics, and Materials Conference. 